\title{
Effects of explaining on children's preference for simpler hypotheses
}

\author{
Caren M. Walker ${ }^{1}$ - Elizabeth Bonawitz ${ }^{2}$ - Tania Lombrozo ${ }^{3}$
}

Published online: 7 February 2017

(C) Psychonomic Society, Inc. 2016

\begin{abstract}
Research suggests that the process of explaining influences causal reasoning by prompting learners to favor hypotheses that offer "good" explanations. One feature of a good explanation is its simplicity. Here, we investigate whether prompting children to generate explanations for observed effects increases the extent to which they favor causal hypotheses that offer simpler explanations, and whether this changes over the course of development. Children aged 4, 5, and 6 years observed several outcomes that could be explained by appeal to a common cause (the simple hypothesis) or two independent causes (the complex hypothesis). We varied whether children were prompted to explain each observation or, in a control condition, to report it. Children were then asked to make additional inferences for which the competing hypotheses generated different predictions. The results revealed developmental differences in the extent to which children favored simpler hypotheses as a basis for further inference in this task: 4-year-olds did not favor the simpler hypothesis in either condition; 5-year-olds favored the simpler hypothesis only when prompted to explain; and 6-year-olds favored the simpler hypothesis whether or not they explained.
\end{abstract}

Keywords Explanation - Cognitive development .

Simplicity $\cdot$ Causal inference

Caren M. Walker

carenwalker@ucsd.edu

1 Department of Psychology, University of California, San Diego, 9500 Gillman Dr., \# 0109, La Jolla, CA 92093-0109, USA

2 Department of Psychology, Rutgers University - Newark, 334 Smith Hall, Newark, NJ 07102, USA

3 Department of Psychology, University of California, Berkeley, 3210 Tolman Hall, Room 1221, Berkeley, CA 94720, USA
A core feature of human intelligence is the drive to explain (Gopnik, 1998). It is no surprise, then, that explanatory conversations are ubiquitous in young children's everyday lives (Newport, Gleitman, \& Gleitman, 1977; Callanan \& Oakes, 1992). Prior work suggests an important role for these early conversations: generating explanations - even when no explicit feedback is provided - influences causal learning in childhood, fostering the growth of domain knowledge (Siegler, 1995; Wellman, 2011; Wellman \& Lagattuta, 2004; Wellman \& Liu, 2007) and encouraging children to go beyond the surface to uncover deeper structure (e.g., Legare \& Lombrozo, 2014; Walker, Lombrozo, Legare, \& Gopnik, 2014). How might explaining generate these effects?

One proposal is that the act of explaining recruits particular constraints on causal reasoning, changing how hypotheses are generated and evaluated (Lombrozo, 2012, 2016; Lombrozo \& Vasilyeva, 2016); Walker et al., 2014; Walker, Lombrozo, Williams, Rafferty, \& Gopnik, 2016; Williams \& Lombrozo, 2010, 2013). Specifically, explaining could encourage learners to privilege those hypotheses that offer the best explanation relative to alternatives, where "better" explanations are those that exhibit explanatory virtues, such as simplicity and breadth. This proposal is closely related to "inference to the best explanation" (Harman, 1965), the idea that an explanation's "loveliness" is used as a cue to its likeliness when comparing among competing hypotheses (Lipton, 2004).

The most compelling evidence that young children engage in a process like inference to the best explanation comes from a study by Bonawitz and Lombrozo (2012), which investigated whether 4- and 5-year-old children favored simpler causal hypotheses. To do so, Bonawitz and Lombrozo introduced children to a novel toy in which different colored chips generated either one effect or two effects when placed in the toy's activator bin: Blue chips activated the toy's fan and light, green chips activated only the fan, and red chips activated only 
the light. Participants were asked to explain the hidden cause of an event in which the toy's fan and light were both activated. Children could appeal to the hypothesis that a blue chip was in the activator bin, which was simpler by virtue of invoking a single common cause, or the hypothesis that multiple chips were in the activator bin (e.g., a green chip and a red chip), which involved multiple independent causes. Across conditions, different children observed different frequencies of each chip type, effectively changing the relative probabilities of these hypotheses.

Overall, children demonstrated a strong preference for simpler explanations. This was especially true when the hypotheses were equally probable, but even when chip frequencies suggested that the complex explanation was more likely, children still favored the simpler explanation more often than warranted by chance. Importantly, however, children's preference for simplicity was not indiscriminate: the baseline preference for simpler explanations was accompanied by a significant effect of base rates, such that children chose the simpler explanation less often when it was less likely to be true. These findings mirror those for adults in a similar diagnostic reasoning task (Lombrozo, 2007).

The findings from Bonawitz and Lombrozo (2012) reveal a systematic preference for simpler causal hypotheses, but leave open whether and how the process of explaining plays a role. If explaining recruits criteria such as simplicity in assessing competing hypotheses, then prompting children to explain could heighten the advantage for simpler hypotheses. Indeed, there is evidence that prompting children to explain heightens sensitivity to the explanatory virtue of scope, which refers to the breadth of data that a given hypothesis can explain. Specifically, Walker, Lombrozo, Williams, Rafferty, and Gopnik (2016) presented 5-year-old children with patterns of evidence that were consistent with two causal hypotheses, and prompted children to either explain or report each piece of evidence. When the competing hypotheses were matched in terms of prior beliefs, but varied in the scope of evidence they accounted for, children prompted to explain were more likely than controls to favor the hypothesis with broader scope. These findings on causal learning in children are matched by findings involving categorization in adults, for whom a prompt to explain similarly leads to privileging hypotheses with broader scope (e.g., Williams \& Lombrozo, 2010, 2013; Williams, Lombrozo, \& Rehder, 2013; see also Chi, DeLeeuw, Chiu, LaVancher, 1994).

In sum, the process of explaining seems to magnify the impact of explanatory preferences in selecting among competing hypotheses (Lombrozo, 2007, 2012; Williams \& Lombrozo, 2010; 2013; Walker et al., 2016, Legare \& Lombrozo, 2014; Walker et al., 2014; Williams, Lombrozo, \& Rehder, 2013). However, no research to date has explored whether the process of explaining likewise affects the explanatory virtue of simplicity, and whether such an influence changes over the course of development.

In the current paper, we explore whether prompts to explain influence children's tendency to privilege simpler hypotheses when engaged in causal inference. To do so, we presented 4-, 5-, and 6-year-olds with an outcome that could be the result of a single common cause (supporting a simple explanation) or two independent causes (the more complex alternative), and we varied whether children were asked to explain or report their observations. Specifically, children were shown two unhealthy plants sampled from different locations in an illustrated garden, and were asked to determine which factors (such as soil type or a broken sprinkler) made the plants sick. The evidence was consistent with a simple, common cause explanation (soil type made both plants sick) and a more complex, independent causes explanation (a broken sprinkler made one of the plants sick, and lack of sun made the other plant sick). In one condition, children were asked to explain each observation (e.g., "Why do you think these plants are sick?"), and in another they were asked to report whether the plants were sick (e.g., "Were these plants healthy or sick?"). As in previous research, reporting was selected as a control task because it shares several features with explanation: it draws the learner's attention to the evidence, it generally matches the amount of time that children spend engaging with each observation between conditions, and it requires children to provide a verbal response in the context of a social interaction (Walker et al., 2014, 2016). Following this training phase, children were presented with a novel garden and asked to predict whether each of four new plants would be healthy or sick, based on the same set of factors. We predicted that children prompted to explain would be more likely than children prompted to report to generalize according to the simple, common cause hypothesis.

We included children between the ages of 4 and 6 for two reasons. First, previous research indicates that by age 4, children have the basic cognitive capacities and domain knowledge required for our task. By age 2, children already possess intuitions about what counts as a satisfying explanation, and, as a result, actively seek causal information in conversations with adults (Frazier, Gelman, \& Wellman, 2009). By age 4, children produce explanations that are domain-appropriate (Schulz, Bonawitz, \& Griffiths, 2007; Hickling \& Wellman, 2001), have abstract, coherent representations of causal relationships in several domains of knowledge (e.g., Carey, 1985; Gelman \& Wellman, 1991; Gopnik \& Meltzoff, 1997; Inagaki \& Hatano, 1993; Perner, 1991), and can use covariation data to form novel inferences based on their observations (e.g., Kushnir, Xu, \& Wellman, 2010). With regard to relevant domain knowledge, there is evidence for substantial variability in children's understanding of plant growth based on their early experiences (e.g., Bang \& Medin, 2010; Coley, 2003; Medin \& Atran, 2004; Ross, Medin, Coley, \& Atran, 2003), 
but by 4.5 years of age, most children have the requisite domain knowledge to reason about possible causes of plant growth, even if the precise causal mechanisms remain underspecified. Specifically, preschoolers hold theory-like conceptions of plant growth, similar to those they hold for the growth of humans and other animals (Hickling \& Gelman, 1995), and 4-year-olds are able to recognize the importance of external natural factors for plant growth, including water and sun (Christidou \& Hatzinikita, 2006).

Second, we anticipated that explaining might influence the manifestation of a preference for simpler hypotheses across this age range. On the one hand, we know that preschoolers and adults show a preference for simpler explanations, even in the absence of prompts to explain (Bonawitz \& Lombrozo, 2012; Lombrozo, 2007). This suggests that a robust preference for simplicity could emerge even in the report condition, rendering a prompt to explain superfluous. On the other hand, there is evidence that prompting younger preschoolers to explain can help them overcome salient but superficial bases for inference, shifting their performance to resemble that of older children on comparable tasks. For example, Walker, Lombrozo, Legare, and Gopnik (2014) presented 3- 5-yearolds with a task in which an internal part could be generalized from one block to another block that was either perceptually similar or causally similar (i.e., it generated the same effect when placed on a novel toy). When prompted to explain, younger children generalized according to causal similarity, which is characteristic of performance by older children in the absence of a prompt (Sobel et al., 2007). Relatedly, Legare, Wellman, and Gelman (2009) found that children in this age range were able to generate accurate explanations for why a person avoided a contaminated food, even though they could not reliably predict which food would be selected until they were older (see also Amsterlaw \& Wellman, 2006; Bartsch \& Wellman, 1989). These findings suggest that prompts to explain could allow an underlying preference for simplicity to emerge by helping children recruit relevant prior knowledge and overcome the allure of salient alternatives, and thus to privilege the "loveliest" hypothesis. If this is the case, we might expect a prompt to explain to heighten a preference for simpler hypotheses in younger children, but be superfluous for older children. Our experiment can test this prediction.

\section{Methods}

\section{Participants}

A total of 180 children participated in Experiment 1, including 60 4-year-olds ( $M=53.0$ months; range 46.0-59.4 months), 605 -year-olds $(M=65.3$ months; range $=59.9-71.8$ months $)$, and 606 -year-olds $(M=76.6$ months; range $=72.8-$ 83.6 months). There were approximately equal numbers of males and females in each age group. Half of the children ( $n$ $=30$ ) in each age group were randomly assigned to each of two between subjects conditions: explain or report. An additional nine participants were tested, but excluded. Five of these children were excluded due to experimenter error, two due to participants' failure to complete the experiment, and two due to caregiver prompting during the experiment. Children were recruited from local preschools and museums in a primarily urban setting. Although we did not collect specific demographic information for each child, demographic information from each recruitment location suggests that the children were predominantly white $(58 \%)$, with a range of ethnicities resembling the local diversity, and that the majority of the population was middle-class (average household income: US\$90,000-140,000 per year).

\section{Materials}

The task involved two gardens (a training version and a test version), each of which was represented by a color illustration, printed on an 8 in $\times 10$ in $(20.3 \mathrm{~cm} \times 25.4 \mathrm{~cm})$ piece of paper. Both training and test gardens were divided into four equal quadrants (see Fig. 1), with two adjacent quadrants depicting red soil and two adjacent quadrants depicting brown soil. Each quadrant also depicted four green patches, indicating the locations of carrots in the ground, and one unique feature: a doghouse, rocks, a broken sprinkler, or a shady tree. The key difference between the training and test gardens was in the placement of the unique features in relation to the soil type. In the training garden, the doghouse and rocks appeared in the quadrants with brown soil, and the broken sprinkler and shady tree appeared in the quadrants with red soil. In the test garden, these soil colors were reversed.

Other materials included a laminated cardboard puppet of a farmer, two laminated cardboard images of "sick" carrots (orange carrots with brown spots), and four black squares of construction paper, designed to occlude each quadrant of the training garden.

\section{Procedure}

Children were tested individually in a small testing room or a quiet corner of a preschool or museum, seated at a table across from the experimenter. During the training phase, the experimenter introduced children to a character named "Mr. Farmer." The experimenter initiated the game, saying, "This is Mr. Farmer, and he is very good at growing plants. He has lots of gardens, with many different kinds of plants. Today, he is going to show you one of his gardens." Indicating that Mr. Farmer is "very good at growing plants" was intended to provide children with the expectation that the majority of plants in his garden are healthy, such that a sick plant would warrant explanation. 


\section{Training Garden}

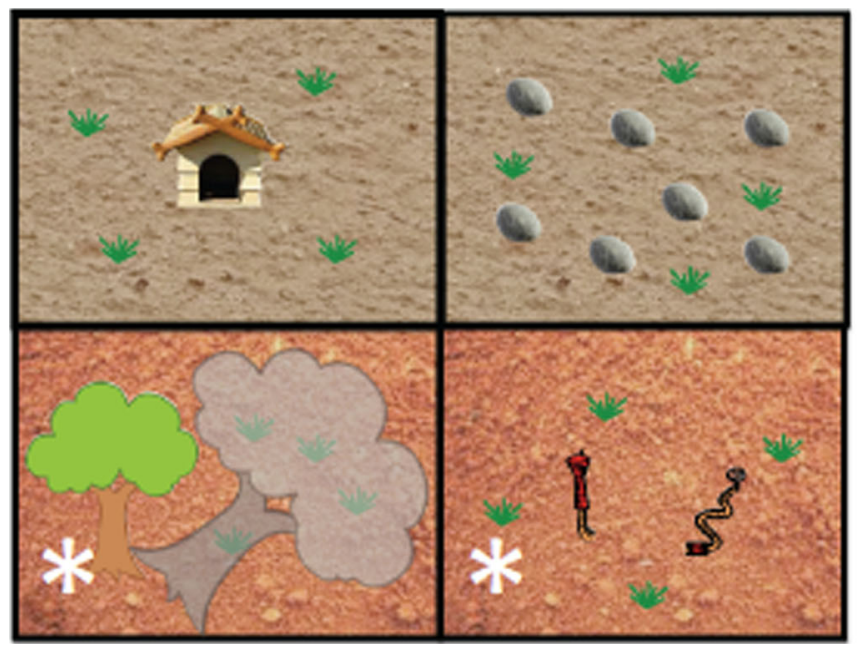

Fig. 1 Exact replication of the garden scenes used in the training and test phases of the experiment. Each quadrant depicts both a soil color (brown or red) and an additional feature (doghouse, rocks, shady tree, or broken sprinkler). The quadrants that contained sick carrots are represented by

The training garden was then presented to the child with squares of black construction paper occluding each of the four quadrants. The experimenter removed each of the black squares, one at a time, introducing the two features in each quadrant. She would say, for example, "In this part of the garden, there is a doghouse and the soil is brown." She introduced each quadrant in this same manner for all four quadrants - labeling both the unique feature (doghouse, rocks, broken sprinkler, shady tree) and the soil type (brown, red). These were explicitly identified and labeled to ensure that children correctly interpreted the illustrations, and to help equate potential differences in the salience of features across children or conditions. The order of presentation of the quadrants was randomized between subjects.

After each of the four quadrants had been uncovered and described, the experimenter provided the following instructions: "This is Mr. Farmer's carrot garden! Some of the carrots are healthy and some of the carrots are sick. I don't know which ones are healthy and which ones are sick, so we have to pick some to find out. Let's try to help Mr. Farmer figure out what makes the carrots healthy or sick." These instructions ensured that all children understood that they were engaged in a causal inference task, with the goal of identifying what makes carrots healthy or sick.

After the instructions, the child was asked to select a carrot to sample from one of the quadrants with red soil (see Fig. 1). When the child selected a carrot, the experimenter placed a picture of a sick carrot in the quadrant, and informed the child that the selected carrot was sick. She then repeated this procedure for the other quadrant with red soil. In both cases, the selected carrot was sick. The observations were therefore

\section{Test Garden}

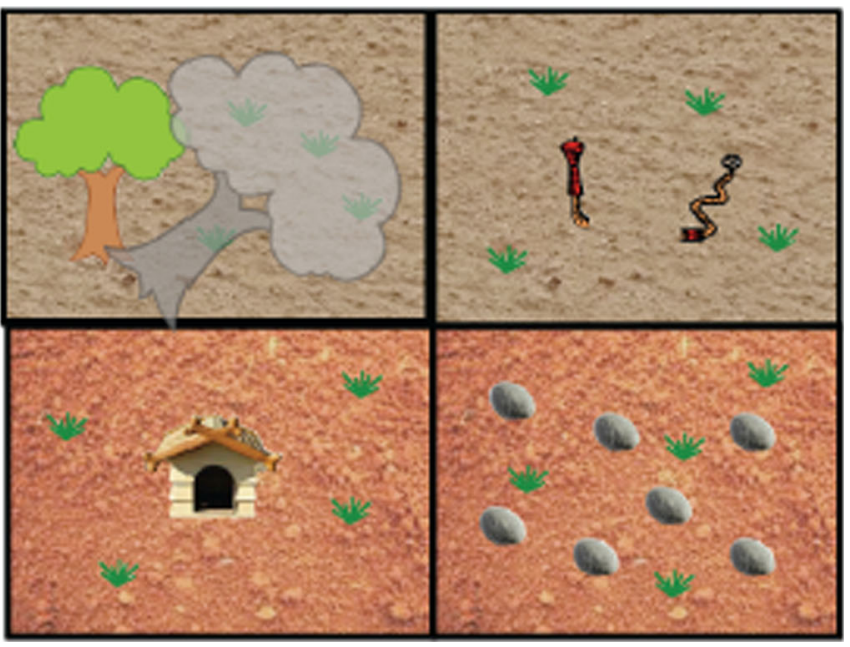

asterisks (asterisks were not present in study stimuli). The test garden reverses the placement of the unique features in relation to the soil type in the training garden

consistent with two key hypotheses: that the two carrots were sick due to a single common cause (red soil), or due to two independent causes (shade in one case, and a broken sprinkler in the other).

The pictures of the sick carrots remained visible in the red soil quadrants of the garden as the experimenter provided one of two prompts (explain or report), depending upon the child's assigned condition. In the explain condition, the experimenter asked: "Now take a look at the garden. Why do you think these two carrots are sick?" In the report condition, the experimenter asked: "Now take a look at the garden. Were these two carrots healthy or sick?" This was the only difference in procedure between conditions. As part of the study design, children were only shown and prompted to explain (or report) cases of sick carrots. Children were not shown healthy carrots, since previous research has found that it is more natural for children to explain anomalies in the data than to explain expected outcomes (e.g., Legare, 2012), and adding additional cases would have complicated the task.

After children provided a response, the training garden was moved to one side of the table, remaining in view. The test garden was then presented, uncovered. The experimenter said: "This is another one of Mr. Farmer's carrot gardens. I am going to ask you some questions about it." She then asked the following question for each of the quadrants in the test garden: "If I pick a carrot from this part of the garden, do you think it will be healthy or sick?" The order of presentation of the two options, "healthy" or "sick," was randomized for each quadrant. These questions were designed to differentiate inferences based on the single common-cause hypothesis (soil type) from the two independent causes hypothesis (lack of 
water and lack of sunlight), since the correlation between soil type and the broken sprinkler and shady tree in the training garden was reversed in the test garden. The pattern of children's predictions would therefore reveal the particular hypothesis (simple, complex, or other) upon which these inferences were based.

\section{Coding}

Children's responses to the prompt for each quadrant in the test garden, "sick" or "healthy," were recorded. Children received 1 point for responding in line with the common-cause hypothesis (soil color) - saying "sick" for the quadrants with red soil and "healthy" for the quadrants with brown soil - and 0 points otherwise. Children could therefore receive a total of 4 points for the test items, one for each quadrant. Total scores closer to 4 thus align with the simple hypothesis, while those closer to 0 align with the complex hypothesis. A second researcher who was naïve to the purpose of the experiment recoded all responses. Inter-rater reliability was very high; the two coders agreed on $98 \%$ of the children's responses.

For children prompted to explain, explanations were also coded. Children's explanations were coded as belonging to one of three categories: (1) simple (i.e., mentioning soil type), (2) complex (i.e., mentioning the broken sprinkler and the shady tree), and (3) other (i.e., mentioning one or a combination of features that did not account for all of the data (e.g., only the sprinkler, the sprinkler and the rocks), providing an irrelevant explanation (e.g., "Because there are cobras around"), or providing no response. A second researcher who was naïve to the purpose of the experiment recoded all responses. Inter-rater reliability was again very high; the two coders agreed on $94 \%$ of the coding of children's explanations.

\section{Results}

Children's responses were analyzed in an ANOVA with the number of predictions consistent with the simple or complex hypothesis $(0-4)$ as the dependent variable, and condition (explain, report) and age group $(4,5,6)$ as independent variables. This analysis revealed a significant effect of age, $F(2,174)=$ $8.37, p<.001, \eta_{\mathrm{p}}{ }^{2}=.088$, which was qualified by a marginally significant interaction between age and condition, $F(2,174)=$ $5.85, p=.055, \eta_{\mathrm{p}}{ }^{2}=.033$. We therefore analyzed the effect of condition separately for each age group (see Fig. 2).

Four year olds' responses did not differ significantly across conditions, $F(1,58)=.345, p=.56$. In fact, their responses did not differ from chance responding (i.e., choosing randomly between "healthy" and sick") for either the report condition $(M=1.9, S D=1.4), t(29)=-0.13, p=.90$, or the explain

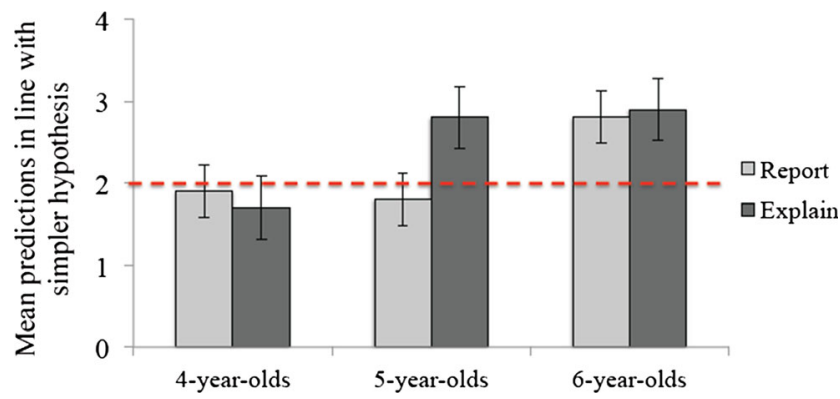

Fig. 2 The graph presents the mean number of predictions (of four) consistent with the simpler, common-cause hypothesis (red soil) for 4-, 5 - and 6 year olds in the report and explain conditions. Six year olds and 5 -year-olds in the explain condition drew inferences that were more consistent with the simpler hypothesis, but 5 -year-olds in the report condition and 5-year-olds in both conditions were not different from the mid-point of 2

condition $(M=1.7, S D=1.2), t(29)=-1.33, p=.19$, suggesting no preference for either hypothesis.

Five year olds, in contrast, showed the expected effect of explanation: they were significantly more likely to make predictions in line with the simpler hypothesis in the explain condition $(M=2.8, S D=1.1)$ than in the report condition $(M=1.8, S D=1.6), F(1,58)=8.24, p=.006, \eta_{\mathrm{p}}{ }^{2}=.12$. Moreover, while their responses in the report condition did not differ from chance, $t(29)=-0.70, p=.49$, their responses were significantly more likely than chance to align with the simple hypothesis in the explain condition, $t(29)=4.0, p<.001$.

Finally, six year olds drew inferences in line with the simpler hypothesis regardless of whether they were prompted to report $(M=2.8, S D=1.6)$ or to explain $(M=2.9, S D=1.5)$, with no difference between conditions, $F(1,58)=.063, p=$ .80 . In both cases, they were significantly more likely than chance to generate predictions consistent with the simpler hypothesis, $t(29)=2.48, p<.02$ (report condition) and $t(29)=$ $3.41, p<.01$ (explain condition).

To better understand the behavior of individual children, we additionally classified participants into five response patterns (see Table 1). Children were classified as "all simple" if all four predictions aligned with the simple hypothesis, and as "all complex" if all four predictions aligned with the complex hypothesis. These patterns correspond to overall prediction scores of 4 and 0 , respectively. We also considered predictions that corresponded to the simple hypothesis and one additional feature (either shade or broken sprinkler, "soil + 1"), responses of "sick" to all four queries ("sick" bias), and responses of "healthy" to all four queries ("healthy" bias). Participants who did not fall into any of these patterns were classified as "other."

The frequency data presented in Table 1 support the statistical analyses reported above, with "all simple" as the modal category for 5-year-olds prompted to explain, as well as for 6-year-olds in either condition. However, the other categories provide additional insight. First, the 
Table 1 Frequency of each pattern of predictions by age group and condition

\begin{tabular}{|c|c|c|c|c|c|c|c|}
\hline Age & Condition & All simple & All complex & Soil + 1 & "Sick" bias & "Healthy" bias & Other \\
\hline 4 & Report & 6 & 7 & 1 & 1 & 2 & 13 \\
\hline 4 & Explain & 3 & 3 & 2 & 1 & 3 & 18 \\
\hline 5 & Report & 7 & 10 & 1 & 4 & 1 & 7 \\
\hline 5 & Explain & 11 & 1 & 4 & 2 & 2 & 10 \\
\hline 6 & Report & 17 & 5 & 0 & 0 & 0 & 8 \\
\hline 6 & Explain & 16 & 4 & 1 & 1 & 1 & 7 \\
\hline
\end{tabular}

modal response for 4-year-olds was "other," suggesting that they lacked either the relevant domain knowledge or domain-general abilities to clearly represent the simple and complex hypotheses as salient bases for inference. By contrast, the 5-year-olds in the report condition were more likely to fall into a defined category, but were no more likely to respond "all simple" than "all complex." This suggests that 5-year-olds succeeded in representing the simple and complex hypotheses as viable bases for inference, but only showed a systematic preference when prompted to explain.

The data in Table 1 also help rule out an alternative interpretation of our main findings: that the prompt to explain led children to provide a more coherent or consistent pattern of responses, without any special role for simplicity. Overall, children who explained were about as likely to provide a mixed ("other") pattern of response as those in the report condition, and only a minority of children across both conditions responded in line with either a "sick" $(n=9)$ or "healthy" $(n=9)$ response bias.

In sum, these results indicate developmental differences in the effect of explanation on children's preference for simpler hypotheses as a basis for further inference. Four year olds failed to show a reliable preference, whether or not they were prompted to explain. Five-year-olds were most sensitive to the prompt, leading them to respond like 6-year-olds. Six-yearolds, in turn, did not need an explanation prompt to reliably draw inferences in line with the simpler hypothesis.

\section{Qualitative analysis of explanations}

Qualitative analyses revealed that the content of children's explanations was systematically related to their later predictions. Of the children who explained (across all age groups), $34 \%$ provided an explanation that referenced the simple hypothesis. These children provided predictions in line with this hypothesis significantly more often than chance $(M=3.3, S D$ $=1.1), t(30)=6.65, p<.0001$, and also more often than the $16 \%$ of children who provided complex explanations $(M=$ $1.1, S D=1.3), t(43)=-6.04, p<.0001$, the $50 \%$ of children who provided other explanations $(M=2.1, S D=1.1), t(74)=$ $-4.78, p<.0001$, and those in the report condition $(M=2.1$,
$S D=1.6), t(119)=-3.79, p<.001$. The $16 \%$ of children who provided complex explanations generated predictions $(M=$ $1.1, S D=1.3$ ) that aligned with the complex hypothesis significantly more often than chance, $t(13)=-2.74, p<.02$, and also more often than those in the report condition $(M=2.1, S D$ $=1.6), t(102)=2.49, p<.02$. Finally, $50 \%$ of children provided other explanations, and their predictions $(M=2.1, S D=$ 1.1) did not differ significantly from those of children in the report condition $(M=2.1, S D=1.6), t(133)=-0.21, p=.84$. Overall, therefore, the content of children's explanations was reliably associated with their later predictions.

Table 2 provides a breakdown of the percentages of each explanation type by age, as well as the mean performance on test trials (out of four) for the corresponding group of children. While small sample sizes prevent statistical comparison, looking at the results as a function of age supports two relevant observations.

First, the majority of 4-year-olds provided explanations coded as "other." This is consistent with their pattern of predictions, which were also frequently classified as "other," and suggests that despite our original assumption, 4-year-olds lacked the relevant domain knowledge to recognize the simple and complex hypotheses as viable alternatives. Without this requisite knowledge, we would not expect a reliable preference for simplicity to emerge, with or without a prompt to explain.

Second, 5- and 6-year-olds differed in the extent to which their predictions tracked their explanations. For 6-year-olds, there was a reliable association: the $50 \%$ of 6 -year-olds who provided simple explanations were significantly more likely to make predictions in line with the simple hypothesis than the 27 $\%$ of six-year-olds who provided complex explanations $(M=3.7$ vs. $M=0.9, t(21)=-7.1, p<.001)$. For 5-year-olds, by contrast, the $37 \%$ who provided simple explanations were no more likely to make predictions in line with this explanation than the $10 \%$ who provided complex explanations $[M=3.0$ vs. $M=2.7, t(12)$ $=-.39, p=.70$ (statistic is based on small sample sizes)]. Nonetheless, the comparison between the explain and report conditions reveals that explaining led 5-year-olds to generate predictions that were more consistent with the simpler hypothesis (see Fig. 2 and Table 1). This suggests that the effects of explanation derived in part from the process of explaining, 
Table 2 Percentage of explanation types and mean total score* by age for children in the explanation condition

\begin{tabular}{llll}
\hline Age & $\begin{array}{l}\text { Simple } \\
\text { explanation }\end{array}$ & $\begin{array}{l}\text { Complex } \\
\text { explanation }\end{array}$ & $\begin{array}{l}\text { Other } \\
\text { explanation }\end{array}$ \\
\hline 4-year-olds & $20 \%(2.3)$ & $7 \%(0.0)$ & $73 \%(1.7)$ \\
5-year-olds & $37 \%(3.0)$ & $10 \%(2.7)$ & $53 \%(2.7)$ \\
6-year-olds & $50 \%(3.7)$ & $27 \%(0.9)$ & $23 \%(2.1)$ \\
\hline
\end{tabular}

*Mean total score appears in parentheses following each percentage

whether or not that process yielded a particular explanation as its outcome. We return to this potentially counterintuitive result in the discussion (see also, Walker et al., 2014, 2016).

\section{Discussion}

The present study had two aims. First, we tested the prediction that engaging in explanation can influence causal inference by encouraging children to favor simpler hypotheses. Second, we explored whether this effect of explanation changes between the ages of 4 and 6.

Overall, we found strong support for our prediction among 5 -year-olds. Five-year-old children who were prompted to explain were significantly more likely than those in the report condition to make predictions in line with a simpler hypothesis. However, we also found a striking pattern of developmental change. Four year olds showed no preference between a simple, common cause hypothesis and a more complex, independent causes alternative in either condition, while 6-yearolds drew inferences in line with the simpler hypothesis in both conditions. We consider each age group in turn.

Our finding that 4-year-olds did not favor the simpler hypothesis contrasts with prior research, which found a reliable preference for simpler explanations in this age group (Bonawitz \& Lombrozo, 2012). We suspect that, contrary to our initial assumptions, 4-year-olds lacked the requisite domain knowledge to engage with the task. Supporting this suspicion, the pattern of explanations provided by 4-year-olds was highly variable and poorly aligned with the intended simple and complex hypotheses. Moreover, past research reveals substantial variability in the development of children's understanding of plant growth, and finds that experience with nature is one important factor (e.g., Bang \& Medin, 2010; Coley, 2003; Medin \& Atran, 2004; Ross, Medin, Coley, \& Atran, 2003). Given that the children included in this study were sampled from primarily urban areas, they may have had limited experience with nature. While this null result with 4-yearolds does not contribute to our initial aims, it highlights the critical role of prior knowledge in explanation as an important topic for further study (see also Walker et al., 2016; Williams \& Lombrozo, 2013).
Our findings with 5-year-olds are consistent with prior work showing that prompts to explain can heighten sensitivity to explanatory virtues and generate more mature patterns of response (e.g., Walker et al., 2016, 2014). However, prior work has focused on other features of explanations, such as their breadth (Walker et al., 2016). Our findings are the first to demonstrate that a prompt to explain can heighten sensitivity to simplicity as a basis for favoring one hypothesis over another. Besides shedding light on the role of explanation in causal learning, this finding supports the idea that simplicity is one of the constraints recruited by explaining.

Interestingly, the effect of explanation on 5-year-olds' inferences occurred independently of the content of their explanations. This is consistent with prior research, which has found that preschoolers who are prompted to explain often show a more sophisticated pattern of responses on later inferences, even when the content of their explanations falls short of the more mature pattern (Walker et al., 2014, 2016). A variety of proposals help make sense of this potentially counterintuitive result (e.g., Wilkenfeld \& Lombrozo, 2015). For example, generating a mediocre or false explanation could help the learner to reject its presuppositions or note gaps in a currently-held theory (e.g., Chi et al., 1994). There is also evidence that explaining encourages other cognitive processes, such as abstraction (e.g., Walker et al., 2014; Walker \& Lombrozo, under review) and comparison (e.g., Edwards, Williams, Lombrozo, \& Gentner, 2013), that could change a learner's representation of the hypothesis space, carrying downstream implications for inference. On these views, engaging in explanation helped 5-year-olds move away from bad explanations and appreciate the structure of the problem, even if it fell short of delivering the simple explanation itself.

Finally, 6-year-olds demonstrated a consistent preference for the simpler hypothesis as a basis for inference, in both the report and explain conditions. There are a few reasons why a prompt to explain may not have changed responses for 6-year-olds. First, it could be that, by age 6 , children spontaneously recruit simplicity as a basis for selecting between competing hypotheses, whether or not they are engaged in explanation. Consistent with this idea, Walker et al. (2014) found that prompting 3- to 5year-old children to explain why blocks activated a machine made the children more likely to privilege causal similarity over perceptual similarity in a subsequent generalization task. However, by age 5 , children began to favor causal similarity even in the control condition, without a prompt to explain (see also Sobel et al., 2007). This suggests that explanation prompts could scaffold inductive preferences that are on the verge of developing, but become superfluous thereafter.

A second possibility is that 6-year-olds are more likely than younger children to engage in explanation spontaneously (i.e., in the absence of a prompt), leading them to privilege simpler hypotheses across conditions by virtue of explaining in both conditions. Consistent with this idea, previous research has 
found that older children are more likely than younger children to produce explanations in response to an ambiguous prompt (Legare \& Lombrozo, 2014). There is also evidence that explanation quality may increase over this developmental period (see Walker et al., 2014). With a high level of baseline explanation for the 6-year-olds, a prompt to explain may not have increased levels of explanation far beyond those found in the report condition.

While both of these two explanations for 6-year-olds' performance are plausible on their own, they are difficult to reconcile with two other results. First, there is evidence from Bonawitz and Lombrozo (2012) that children favor simpler hypotheses by age 4 , even when the inference task is not preceded by explicit prompts to explain. This suggests that a preference for simplicity is not what develops between 4 and 6. Second, explanation prompts have been shown to impact learning in older children and in adulthood (e.g., Fonseca \& Chi, 2010; Lombrozo, 2012), suggesting that levels of spontaneous explanation are rarely high enough to generate a true ceiling effect. In light of these results, what can account for the shift we observe between the ages of 5 and 6 ?

We think the data reported in Table 1 provide an important hint: the modal response pattern for 5-year-olds in the report condition was to draw inferences in line with the complex hypothesis. This hypothesis identifies two causes of poor plant growth that are likely to be salient for children, both in light of their prior knowledge and the context of the task: poor light (due to shade) and insufficient water (due to the broken sprinkler). By contrast, children are unlikely to have strong beliefs about which color of soil could affect plant growth, potentially making the simple hypothesis less salient. If this is the case, then the more salient complex hypothesis might have competed against the simpler hypothesis for both 5- and 6-year-old children, but whereas 5-year-olds required the help of an explanation prompt to overcome it in favor of the simpler alternative, 6-year-olds did not.

This explanation for developmental change mirrors the findings from Walker et al. (2014), referenced above. In those studies, an explanation helped younger children overcome a tendency to respond on the basis of salient properties, and thus revealed an underlying ability to privilege alternative bases for inference: either causal similarity or common category membership. In the present case, the idea is that explanation did not influence 5-year-old children's subsequent inferences by introducing a preference for simpler explanations that they did not already possess, but instead that it allowed them to effectively execute this preference despite a compelling alternative. And for 6-year-olds, explanation was not ineffective because spontaneous explanation was at ceiling, but rather because all children were in a better position to resist the allure of the salient, two-cause hypothesis and seriously consider the simpler alternative, just as 6-year-olds are also in a better position to overcome salient, perceptual properties in favor of causal properties or category membership as a basis for inference.

If this interpretation is correct, our findings raise an intriguing possibility: that there exist certain periods during development, or "windows of opportunity," in which explaining is a particularly effective scaffold for learning, helping children overcome less reflective response tendencies and allowing alternative bases for inference to emerge. The boundaries of these windows likely depend upon both existing content knowledge and reasoning capacities in a given domain. In the current case, we speculate that 4-year-olds lacked the requisite domain knowledge to benefit from a prompt to explain, while 6-year-olds did not need an explanation prompt to override the pull of a salient hypothesis.

These results shed light on the mechanisms by which explanation informs and constrains causal learning in early childhood. There is growing evidence that the acquisition of early causal knowledge is supported by learning mechanisms that allow learners to integrate new observations with prior beliefs (e.g., Gopnik et al., 2004; Griffiths, Sobel, Tenenbaum \& Gopnik, 2011). Moreover, there is evidence that even young children are fairly sophisticated probabilistic reasoners (e.g., Gopnik et al., 2004; Griffiths, Sobel, Tenenbaum \& Gopnik, 2011; Schulz, Bonawitz, \& Griffiths, 2007). However, observations are often compatible with multiple hypotheses, and probabilistic information is sometimes unavailable or opaque. In such cases, explanatory considerations - such as simplicity - could play an especially important role in helping children choose between competing hypotheses. The presents results suggest that, by engaging in explanation, these explanatory considerations are more likely to emerge as a basis for constraining inductive inference (see also Walker et al., 2016; Lombrozo, 2012).

If this account is right, it will be important to better articulate the nature of explanatory virtues, such a simplicity and scope. Here, we operationalized simplicity in line with prior work (Bonawitz \& Lombrozo, 2012; Lombrozo, 2007) by contrasting a single, common cause hypothesis against an alternative that appealed to two independent causes. Research with adults suggests that it is not the number of causes in the explanation per se that defines simplicity, but instead the number of causes that are themselves simply posited or unexplained (Pacer \& Lombrozo, 2015). This view of simplicity is consistent with the present results, but it is also possible that our findings reflect some role for scope. If learners only considered (simple) single-cause hypotheses (soil OR broken sprinkler OR shade), they may have favored the soil hypothesis not on the grounds of simplicity but because it accounted for both observations rather than only one that is, it had broader scope. Other examples with adults involve a similar interplay between simplicity and scope 
(Williams, Lombrozo, \& Rehder, 2013). Considering how different explanatory considerations interact in different learning contexts, for different knowledge domains, and over different developmental periods, will be a particularly important avenue for future work.

It is also important to consider the interplay between simplicity and information about probability. Work with adults reveals that simplicity is only used as a basis for selecting between competing explanations when probability information is unavailable or opaque. In particular, both Lagnado (1994) and Lombrozo (2007) found that adults favored a two-cause explanation over a common-cause explanation when it was explicitly identified as more likely. However, Lombrozo (2007) and Pacer and Lombrozo (2015) instead considered cases that more closely mirror real-world situations: participants were presented with many examples from which they could extract the base rates of various causes, but were never explicitly told which explanation was most likely. Under these conditions, explanation choices were influenced by both simplicity and probability. This suggests that explanatory considerations may not be equally influential across contexts, but instead emerge as a basis for inference in the absence of more reliable and readily available cues to probability.

Finally, although explaining appears to impact which hypotheses are privileged, it is not clear that engaging in explanation always leads to better causal learning (e.g., Walker et al., 2016). Explaining is certainly beneficial in many cases: in young children, it has been shown to promote number conservation (Siegler, 1995) and an understanding of balance (Pine \& Siegler, 2003) and false belief (Wellman \& Lagattuta, 2004; Amstertlaw \& Wellman, 2006). But in some cases, the process of explaining may also lead a learner to ignore potentially relevant information (e.g., Walker et al., 2014; Legare \& Lombrozo, 2014), including counterexamples to broad patterns (Williams, Lombrozo, \& Rehder, 2013). In fact, there are several recorded instances in which explanation causes a departure from normative causal reasoning (e.g., Kuhn \& Katz, 2009; Walker et al., 2016). The current results cannot speak to the normativity of children's performance (since there was no independent basis for favoring the oneor two-cause hypothesis), but this raises important questions for future work.

In sum, there is growing evidence that the process of explaining influences causal learning and inference in young children. Both age and prior knowledge may play an important role in this process, defining a window of opportunity in which engaging in explanation has the greatest impact. We propose that, by engaging in explanation, even young learners are more likely to privilege those hypotheses that offer "lovely" explanations: those that appeal to explanatory virtues, such as simplicity.
Acknowledgments Research was funded by grants from the National Science Foundation (grant DRL-1056712) and a James S. McDonnell Foundation Scholar Award in Understanding Human Cognition to T. Lombrozo and the American Psychological Foundation (Elizabeth Munsterberg Koppitz) to C. Walker. The authors thank the parents and children who participated in this study, as well as the University of California, Berkeley Early Childhood Education Centers, the Lawrence Hall of Science, and The Childhood Creativity Center at the Bay Area Discovery Museum. We are grateful to Brian Edwards for his contribution in developing these methods. Finally, we thank Shin Er Teh, Avenee Nulkar, Christine Rickansrud, Sarah Tencher, and especially Rotem Aboody for their assistance with data collection.

\section{References}

Amsterlaw, J., \& Wellman, H. (2006). Theories of mind in transition: A microgenetic study of the development of false belief understanding. Journal of Cognition and Development, 7, 139-172.

Bang, M., \& Medin, D. (2010). Cultural processes in science education: Supporting the navigation of multiple epistemologies. Science Education, 94(6), 1008-1026.

Bartsch, K., \& Wellman, H. (1989). Young children's attribution of action to beliefs and desires. Child Development, 60, 946-964.

Bonawitz, E. B., \& Lombrozo, T. (2012). Occam's rattle: Children's use of simplicity and probability to constrain inference. Developmental Psychology, 48(4), 1156-1164.

Callanan, M. A., \& Oakes, L. M. (1992). Preschoolers' questions and parents' explanations: Causal thinking in everyday activity. Cognitive Development, 7, 213-233.

Carey, S. (1985). Conceptual change in childhood. Cambridge: MIT Press/Bradford Books.

Chi, M. T. H., de Leeuw, N., Chiu, M. H., \& LaVancher, C. (1994). Eliciting self-explanations improves understanding. Cognitive Science, 18, 439-477.

Christidou, V., \& Hatzinikita, V. (2006). Preschool children's explanations of plant growth and rain formation: A comparative analysis. Research in Science Education, 36(3), 187-210.

Coley, J. D. (2003). On the importance of comparative research: The case of folkbiology. Child Development, 71(1), 82-90.

Edwards, B. J., Williams, J. J., \& Lombrozo, T. (2013). Effects of explanation and comparison on category learning In M. Knauff, M. Pauen, N. Sebanz, \& I. Wachsmuth (Eds.), Proceedings of the 35th Annual Conference of the Cognitive Science Society (pp. 406-411). Austin, TX: Cognitive Science Society.

Fonseca, B., \& Chi, M. T. H. (2010). The self-explanation effect: A constructive learning activity. In R. Mayer \& P. Alexander (Eds.), The Handbook of Research on Learning and Instruction. New York: Routledge Press. doi:10.4324/9780203839089

Frazier, B. N., Gelman, S. A., \& Wellman, H. M. (2009). Preschoolers' search for explanatory information within adult-child conversation. Child Development, 80, 1592-1611. doi:10.1111/j.14678624.2009.01356.x

Gelman, S. A., \& Wellman, H. M. (1991). Insides and essences: Early understandings of the non-obvious. Cognition, 38(3), 213-244. doi:10.1016/0010-0277(91)90007-Q

Gopnik, A. (1998). Explanation as Orgasm. Minds and Machines, 8(1), $101-118$

Gopnik, A., Glymour, C., Sobel, D., Schulz, L., Kushnir, T., \& Danks, D. (2004). A theory of causal learning in children: Causal maps and Bayes nets. Psychological Review, 111, 1-31. doi:10.1037/0033295X.111.1.3

Gopnik, A., \& Meltzoff, A. N. (1997). Words, thoughts and theories. Cambridge, MA: MIT Press. doi:10.5860/CHOICE.34-6547 
Griffiths, T. L., Sobel, D., Tenenbaum, J. B., \& Gopnik, A. (2011). Bayes and blickets: Effects of knowledge on causal induction in children and adults. Cognitive Science, 35, 1407-1455. doi:10.1111/j.15516709.2011.01203.x

Harman, G. H. (1965). The inference to the best explanation. Philosophical Review 74 (1):88-95.

Hickling, A. K., \& Gelman, S. A. (1995). How does your garden grow? Early conceptualization of seeds and their place in the plant growth cycle. Child Development, 66(3), 856-876.

Hickling, A. K., \& Wellman, H. M. (2001). The emergence of children's causal explanations and theories: Evidence from everyday conversation. Developmental Psychology, 37, 668-683. doi:10.1037/00121649.37.5.668

Inagaki, K., \& Hatano, G. (1993). Young children's understanding of the mind-body distinction. Child Development, 64, 1534-1549. doi: $10.2307 / 1131551$

Kuhn, D., \& Katz, J. (2009). Are self-explanations always beneficial? Journal of Experimental Child Psychology, 103, 386-394. doi:10.1016/j.jecp.2009.03.003

Kushnir, T., Xu, F., \& Wellman, H. M. (2010). Young children use statistical sampling to infer the preferences of others. Psychological Science, 21, 1134-1140. doi:10.1177/0956797610376652

Lagnado, D. (1994). The psychology of explanation: A Bayesian approach. Masters Thesis. Schools of Psychology and Computer Science, University of Birmingham.

Legare, C. H. (2012). Exploring explanation: Explaining inconsistent evidence informs exploratory hypothesis-testing behavior in young children. Child Development, 83, 173-185.

Legare, C. H., \& Lombrozo, T. (2014). Selective effects of explanation on learning during early childhood. Experimental Child Psychology, 126, 198-212.

Legare, C. H., Wellman, H. M., \& Gelman, S. (2009). Evidence for an explanation advantage in naïve biological reasoning. Cognitive Psychology, 58(2), 177-194.

Lipton, P. (2004). Inference to the best explanation. Psychology Press.

Lombrozo, T. (2007). Simplicity and probability in causal explanation. Cognitive Psychology, 55, 232-257.

Lombrozo, T. (2012). Explanation and abductive inference. In K. J. Holyoak \& R. G. Morrison (Eds.), Oxford Handbook of Thinking and Reasoning (pp. 260-276). Oxford: Oxford University Press.

Lombrozo, T. (2016). Explanation. In J. Sytsma \& W. Buckwalter (Eds.), Blackwell Companion to Experimental Philosophy (pp. 491-503). Blackwell. doi:10.1002/9781118661666.ch34

Lombrozo, T. \& Vasilyeva, N. (2016). Causal explanation. In M. Waldmann (Ed.), Oxford Handbook of Causal Reasoning. Oxford, UK: Oxford University Press.

Medin, D., \& Atran, S. (2004). The native mind: Biological categorization and reasoning in development and across cultures. Psychological Review, 111(4), 960-983.

Newport, E., Gleitman, H., \& Gleitman, L. (1977). Mother, I'd rather do it myself: Some effects and non-effects of maternal speech style. Talking to children: Language input and acquisition, 109-149.
Pacer, M. \& Lombrozo, T. (2015). Occam's razor cuts to the root: Simplicity in causal explanation. Manuscript under review.

Perner, J. (1991). Understanding the representational mind. Cambridge: MIT Press.

Pine, K. J. \& Siegler, R. S. (2003). The role of explanatory activity in increasing the generality of thinking. Paper presented at the biennial meeting of the Society for Research in Cognitive Development, Tampa, FL.

Ross, N., Medin, D., Coley, J. D., \& Atran, S. (2003). Cultural and experiential differences in the development of folkbiological induction. Cognitive Development, 18(1), 25-47.

Schulz, L. E., Bonawitz, E. B., \& Griffiths, T. L. (2007). Can being scared make your tummy ache? Naive theories, ambiguous evidence and preschoolers' causal inferences. Developmental Psychology, 43, 1124-1139. doi:10.1037/0012-1649.43.5.1124

Siegler, R. S. (1995). How does change occur: A microgenetic study of number conservation. Cognitive Psychology, 28, 225-273.

Sobel, D. M., Yoachim, C. M., Gopnik, A., Meltzoff, A. N., \& Blumenthal, E. J. (2007). The blicket within: Preschoolers' inferences about insides and causes. Journal of Cognition and Development, 8, 159-182.

Walker, C. M., Lombrozo, T., Legare, C. H., \& Gopnik, A. (2014). Explanation prompts children to privilege inductively rich properties. Cognition, 133(2), 343-357.

Walker, C. M., Lombrozo, T., Williams, J. J., Rafferty, A., \& Gopnik, A. (2016). Explaining constrains causal learning in childhood. Child Development.

Wellman, H. M. (2011). Reinvigorating explanations for the study of early cognitive development. Child Development Perspectives, 5(1), 33-38.

Wellman, H. M., \& Lagattuta, K. H. (2004). Theory of mind for learning and teaching: The nature and role of explanation. Cognitive Development, 19, 479-497.

Wellman, H. M. \& Liu, D. (2007). Causal reasoning as informed by the early development of explanations. In L. Schulz, \& A. Gopnik (Eds.), Causal Learning: Psychology, Philosophy, \& Computation (pp. 261-279). doi: 10.1093/acprof:oso/ 9780195176803.001.0001

Wilkenfeld, D. A. \& Lombrozo, T. (2015). Inference to the best explanation (IBE) versus explaining for the best inference (EBI). Science and Education, 1-19.

Williams, J. J., \& Lombrozo, T. (2010). The role of explanation in discovery and generalization: Evidence from category learning. Cognitive Science, 34, 776-806.

Williams, J. J., \& Lombrozo, T. (2013). Explanation and prior knowledge interact to guide learning. Cognitive Psychology, 66(1), 55-84.

Williams, J. J., Lombrozo, T., \& Rehder, B. (2013). The hazards of explanation: Overgeneralization in the face of exceptions. Journal of Experimental Psychology: General, Advanced online publication. doi:10.1037/a0030996 